\title{
MATRIX VALUED ORTHOGONAL POLYNOMIALS ARISING FROM GROUP REPRESENTATION THEORY AND A FAMILY OF QUASI-BIRTH-AND-DEATH PROCESSES*
}

\author{
F. ALBERTO GRÜNBAUM ${ }^{\dagger}$ AND MANUEL D. DE LA IGLESIA $\ddagger$
}

\begin{abstract}
We consider a family of matrix valued orthogonal polynomials obtained by Pacharoni and Tirao in connection with spherical functions for the pair $(\mathrm{SU}(N+1), \mathrm{U}(N))$; see [I. Pacharoni and J. A. Tirao, Constr. Approx., 25 (2007), pp. 177-192]. After an appropriate conjugation, we obtain a new family of matrix valued orthogonal polynomials where the corresponding block Jacobi matrix is stochastic and has special probabilistic properties. This gives a highly nontrivial example of a nonhomogeneous quasi-birth-and-death process for which we can explicitly compute its "nstep transition probability matrix" and its invariant distribution. The richness of the mathematical structures involved here allows us to give these explicit results for a several parameter family of quasi-birth-and-death processes with an arbitrary (finite) number of phases. Some of these results are plotted to show the effect that choices of the parameter values have on the invariant distribution.
\end{abstract}

Key words. matrix valued orthogonal polynomials, Markov chains, block tridiagonal transition matrix, quasi-birth-and-death processes

AMS subject classifications. 60J10, 42C05

DOI. $10.1137 / 070697604$

1. Purpose and contents of the paper. The aim of this paper is to tie together two subjects that have received quite a bit of attention recently. We will not give a detailed explanation of either one of them, since this would require too much space and it has been done properly in the literature already. Besides, since these two subjects require rather different backgrounds, an ab-initio exposition would be a formidable task. To compensate for this we give a brief historical view of how these topics developed and then combine them at the appropriate point. The contents of this paper can be divided into three parts.

A first part gives a brief account of the subjects that are going to play a role in this paper. The introduction contains some historical developments tying the moment problem with spectral theory and a quick look at birth-and-death processes, including the appearance of the appropriate orthogonal polynomials. Section 3 reviews very briefly Kreı̆n's theory of matrix valued orthogonal polynomials and discusses the first example relevant to our considerations. Section 4 gives a minimal description of the class of Markov chains known as quasi-birth-and-death processes and talks about the very natural connection between this and the previous section.

The second part introduces the family of examples arising from group representation theory that we are going to use in this paper. Section 5 gives a guide to the literature on matrix valued spherical functions aimed at showing how the examples discussed in section 6 arose. Section 6 gives the bare-bones details of the extensive

${ }^{*}$ Received by the editors July 18, 2007; accepted for publication (in revised form) by H. J. Woerdeman March 14, 2008; published electronically July 2, 2008.

http://www.siam.org/journals/simax/30-2/69760.html

$\dagger$ Department of Mathematics, University of California, Berkeley, Berkeley, CA 94720 (grunbaum@ math.berkeley.edu). This author's work was partially supported by NSF grant DMS-0603901.

${ }_{\ddagger}$ Departamento de Análisis Matemático, Universidad de Sevilla, Apdo (P.O. BOX) 1160, 41080 Sevilla, Spain (mdi29@us.es). This author's work was partially supported by D.G.E.S., ref. BFM2003-06335-C03-01, FQM-262 P06-FQM-01735, FQM-481 (Junta de Andalucía). 
work carried out in [30] in the case of the complex projective space. Section 7 shows how to conjugate the weight matrix from [30] to obtain a family of matrix valued orthogonal polynomials with extra probabilistic properties.

The third part concentrates on the probabilistic aspects of our family of examples. Section 8 deals with a number of issues of probabilistic nature and displays the network associated with our examples. In particular we find explicit expressions for the invariant distribution. Section 9 gives graphical displays of some results obtained from the exact formulas in the previous sections. The goal here is to show that by varying the parameters afforded by the group theoretical situation one can obtain quite a range of different probabilistic behaviors. Finally, section 10 gives a summary of the results of the paper and the challenges that lie ahead.

2. Introduction. The classical Hausdorff moment problem, that of determining a measure $d \psi(x)$ in the interval $[-1,1]$ from its moments

$$
\sigma_{n}=\int_{-1}^{1} x^{n} d \psi(x)
$$

originated in very concrete problems at the end of the 19th century and was discussed by people such as Chebyshev, Markov, and Stieltjes. In the hands of Weyl and a few others, this showed the far reaching power of the modern theory of functional analysis in the early part of the 20th century. The main ingredient here is to connect this problem with the spectral theory of a second order difference operator (built from the moments $\sigma_{n}$ ) acting on functions defined on the nonnegative integers. The moments in question determine (up to scalars) a family of polynomials $\left\{Q_{n}(x)\right\}_{n \geq 0}$, and these polynomials are the eigenfunctions of the second order difference operator alluded to above. In the appropriate Hilbert space this operator is symmetric, and the problem of finding $d \psi(x)$ is the problem of finding self-adjoint extensions of this symmetric operator. Under certain conditions there is a unique such extension and thus a unique solution to the moment problem we started from, but at any rate any extension gives a measure $d \psi(x)$ that makes the polynomials orthogonal with respect to each other.

To get closer to our subject we need a few more ingredients. One of them is given in the rest of this section, and the other two in sections 3 and 4.

The presence of a second order difference operator acting on the space of functions defined on the nonnegative integers, i.e., a semi-infinite tridiagonal matrix, makes it natural to think of a very special kind of Markov chain on the space of nonnegative integers. These are the so-called birth-and-death processes where at each discrete unit of time a transition is allowed from state $i$ to state $j$ with probability $P_{i j}$ and we put $P_{i j}=0$ if $|i-j|>1$. The one-step transition probability matrix is given by

$$
P=\left(\begin{array}{ccccc}
r_{0} & p_{0} & & & \\
q_{1} & r_{1} & p_{1} & & \\
& q_{2} & r_{2} & p_{2} & \\
& & \ddots & \ddots & \ddots
\end{array}\right) .
$$

We will assume that $p_{j}>0, q_{j+1}>0$, and $r_{j} \geq 0$ for $j \geq 0$. We also assume $p_{j}+r_{j}+q_{j}=1$ for $j \geq 1$ and by putting $p_{0}+r_{0} \leq 1$ we allow for the state $j=0$ to be an absorbing state (with probability $1-p_{0}-r_{0}$ ). Some of these conditions can be relaxed. 
The problem here is to obtain an expression for the so-called "n-step transition probability matrix," giving the probability of going between any two states in $n$ steps. By making use of the ideas mentioned above, i.e., by bringing in an appropriate Hilbert space and applying then the spectral theorem, Karlin and McGregor [20] obtained a neat representation formula for the quantity of interest, as recalled below.

If one introduces the polynomials $\left\{Q_{n}(x)\right\}_{n \geq 0}$ by the conditions $Q_{-1}(x)=0$, $Q_{0}(x)=1$ and by using the notation

$$
\phi=\left(\begin{array}{c}
Q_{0}(x) \\
Q_{1}(x) \\
\vdots
\end{array}\right),
$$

one insists on the recursion relation

$$
P \phi=x \phi,
$$

it is possible to prove the existence of a unique measure $d \psi(x)$ supported in $[-1,1]$ such that

$$
\int_{-1}^{1} Q_{i}(x) Q_{j}(x) d \psi(x) / \int_{-1}^{1} Q_{j}(x)^{2} d \psi(x)=\delta_{i j}
$$

and one gets the Karlin-McGregor representation formula

$$
P_{i j}^{n}=\int_{-1}^{1} x^{n} Q_{i}(x) Q_{j}(x) d \psi(x) / \int_{-1}^{1} Q_{j}(x)^{2} d \psi(x) .
$$

If time is taken to be continuous, as it is done in other papers by Karlin and McGregor, then this formula and the matrix $P$ suffer only cosmetic changes.

It is interesting to notice that this seminal paper of Karlin and McGregor refers both to the standard text on the moment problem at the time [33], as well as to the fact that Feller and McKean had already recognized the relevance of the Hilbert space setup in the study of diffusion processes; see $[7,26]$. One can mention other papers, such as $[8,17,19,25]$, where similar ideas were at play.

The last section of [20] deals with the case of a finite state space and the case when the nonnegative integers are replaced by the set of all integers. Since one is using a very powerful tool such as the spectral theorem it is clear that an adaptation of the ideas from birth-and-death processes will work here too. In the case of the integers, one is dealing with a state space with two singular points (one at each end of the line), and in this case Weyl and others had already found the correct tool: one replaces the spectral measure $d \psi(x)$ by a $2 \times 2$ nonnegative matrix. The paper of Karlin and McGregor concludes with the explicit computation of this matrix in the case of the doubly infinite random walk. The general formula is given in expression (12) of [20] for the case of discrete time and also in (6.8) of [18] for continuous time.

The representation formula given above is of intrinsic interest: the computation of the left-hand side of (2.2) for fixed $i, j$ and arbitrary values of $n$ involves all of the entries of (2.1). However, if $d \psi(x)$ is known, then the right-hand side of (2.2) gives a way of computing this quantity using only a fixed number of entries of (2.1).

The applicability of (2.2) depends to a large extent on our ability to obtain useful expressions for the orthogonal polynomials and the orthogonality measure associated 
with $P$. If one looks around in the literature one discovers that the number of cases where this is possible is rather small.

We close this section by showing how one can compute in the case of a stochastic matrix $P$ its invariant (stationary) distribution, i.e., the (unique up to scalars) row vector

$$
\boldsymbol{\pi}=\left(\pi_{0}, \pi_{1}, \pi_{2}, \ldots\right)
$$

such that

$$
\pi P=\pi
$$

Recall that a matrix $P$ with nonnegative entries is called stochastic if the sum of the elements in any row equals unity.

We first obtain, using $r_{0}+p_{0}=1$, that $\pi_{1}=\pi_{0} p_{0} / q_{1}$. Then one proves by induction that for $i \geq 1$ we have

$$
\pi_{i}=\pi_{0}\left(p_{0} p_{1} \ldots p_{i-1}\right) /\left(q_{1} q_{2} \ldots q_{i}\right) .
$$

This has the consequence that

$$
\pi_{i+1} / \pi_{i}=p_{i} / q_{i+1}
$$

Now for $i \geq 0$ we have

$$
x Q_{i}(x)=p_{i} Q_{i+1}(x)+r_{i} Q_{i}(x)+q_{i} Q_{i-1}(x)
$$

with $q_{0}=0$. Integrating this after multiplication by $Q_{i+1}$ or $Q_{i-1}$ gives

$$
\int_{-1}^{1} x Q_{i}(x) Q_{i+1}(x) d \psi(x)=p_{i} \int_{-1}^{1} Q_{i+1}^{2}(x) d \psi(x)=q_{i+1} \int_{-1}^{1} Q_{i}^{2}(x) d \psi(x) .
$$

Combining these two results we get that the ratio of the two integrals above is given by the common value

$$
q_{i+1} / p_{i}=\pi_{i} / \pi_{i+1}
$$

The moral of this is that the solution to $\pi P=\pi$ can be computed (up to a common multiplicative scalar) either from the matrix $P$ itself or from the knowledge of the integrals

$$
\int_{-1}^{1} Q_{i}^{2}(x) d \psi(x)
$$

In particular if we have an homogeneous birth-and-death process where $p_{i}=p$ and $q_{i}=q$ independently of the value of $i$, then we have that the components of $\boldsymbol{\pi}$ are given by $\pi_{i}=\pi_{0}(p / q)^{i}, i \geq 0$.

3. Matrix valued orthogonal polynomials. We need two more characters to be able to start our tale. The first one is the theory of matrix valued orthogonal polynomials, whose bare-bones development is given in two papers by Krel̆n [21, 22]. There is no written account of the motivation that led Kreı̆n to this theory, but one can easily see the connection with the spectral theory of difference operators on the integers. This is very nicely discussed in the book by Berezans'kiı [2]. In fact the study 
of the classical second order difference operator on the integers is done in detail in [2] and may constitute the first example of the theory of Kreln, where the polynomials and their orthogonality weight matrix $W(x)$ are both explicitly given. This is, of course, a special case of the matrix that appears in the last section of [20] for general values of $p$ and $q(p+q=1)$.

We give now a brief account of Krĕn's theory.

Given a positive definite matrix valued measurable weight function $W=W(x)$ with finite moments we can consider the skew symmetric bilinear form defined for any pair of matrix valued polynomial functions $P(x)$ and $Q(x)$ by the numerical matrix

$$
(P, Q)=(P, Q)_{W}=\int_{\mathbb{R}} P(x) W(x) Q^{*}(x) d x,
$$

where $Q^{*}(x)$ denotes the conjugate transpose of $Q(x)$. We define the matrix valued norm of $P$ by

$$
\|P\|^{2}=(P, P)_{W} .
$$

One can also deal with a more general weight matrix $W(x)$; see [4].

This leads, using the Gram-Schmidt process, to the existence of a sequence of matrix valued orthogonal polynomials with nonsingular leading coefficients. Given an orthogonal sequence $\left\{Q_{n}(x)\right\}_{n \geq 0}$ one gets a three term recursion relation

$$
x Q_{n}(x)=A_{n} Q_{n+1}(x)+B_{n} Q_{n}(x)+C_{n} Q_{n-1}(x),
$$

where $A_{n}$ is nonsingular. We will denote by $\mathcal{L}$ the corresponding Jacobi matrix, defined by the following block tridiagonal semi-infinite matrix:

$$
\mathcal{L}=\left(\begin{array}{ccccc}
B_{0} & A_{0} & & & \\
C_{1} & B_{1} & A_{1} & & \\
& C_{2} & B_{2} & A_{2} & \\
& & \ddots & \ddots & \ddots
\end{array}\right)
$$

Using the notation

$$
\Phi=\left(\begin{array}{c}
Q_{0}(x) \\
Q_{1}(x) \\
\vdots
\end{array}\right)
$$

the relation (3.2) becomes

$$
\mathcal{L} \Phi=x \Phi
$$

We will reserve the symbol $P$ for the case where $\mathcal{L}$ becomes a one-step transition probability matrix, thought of as a scalar matrix. The corresponding Markov chain (to appear in section 4) will have a state space that is more complicated than the set $\{0,1,2, \ldots\}$ corresponding to a birth-and-death process featured in section 2 .

In the scalar case, concrete examples of orthogonal polynomials, including explicit formulas for them as well as their orthogonality measure preceded the development of any general theory. Prominent examples are the Hermite, Laguerre, and Jacobi 
polynomials. These examples arose from concrete problems in the eighteenth and nineteenth centuries and played a fundamental role, in the hands of Schrödinger, in the development of quantum mechanics around 1925.

The situation in the matrix valued case is entirely different: the general theory just described above came first. Until a few years ago, it may be that the only nontrivial example was the one included in Berezans'kiu's book [2], alluded to above and recalled below for the benefit of the reader.

Consider the block tridiagonal matrix

$$
\mathcal{L}=\left(\begin{array}{ccccc}
B_{0} & I & & & \\
C_{1} & B_{1} & I & & \\
& C_{2} & B_{2} & I & \\
& & \ddots & \ddots & \ddots
\end{array}\right)
$$

with $2 \times 2$ blocks given as follows:

$$
\begin{aligned}
B_{0} & =\frac{1}{2}\left(\begin{array}{ll}
0 & 1 \\
1 & 0
\end{array}\right), \quad B_{n}=0 \quad \text { if } n \geq 1 \\
C_{n} & =\frac{1}{4} I \quad \text { if } n \geq 1
\end{aligned}
$$

where $I$ stands for the identity matrix. In this case one can compute explicitly the matrix valued polynomials $\left\{Q_{n}(x)\right\}_{n \geq 0}$ given by

$$
x Q_{n}(x)=Q_{n+1}(x)+B_{n} Q_{n}(x)+C_{n} Q_{n-1}(x), \quad Q_{-1}(x)=0, \quad Q_{0}(x)=I .
$$

One gets

$$
Q_{n}(x)=\frac{1}{2^{n}}\left(\begin{array}{cc}
U_{n}(x) & -U_{n-1}(x) \\
-U_{n-1}(x) & U_{n}(x)
\end{array}\right),
$$

where $U_{n}(x)$ are the Chebyshev polynomials of the second kind.

The orthogonality measure is read off from the identity

$$
\frac{4^{i}}{\pi} \int_{-1}^{1} Q_{i}(x) \frac{1}{\sqrt{1-x^{2}}}\left(\begin{array}{ll}
1 & x \\
x & 1
\end{array}\right) Q_{j}^{*}(x) d x=\delta_{i j} I .
$$

Proceeding as in $[3,10,20]$ one obtains a Karlin-McGregor representation. We get, for $n=0,1,2, \ldots$,

$$
\mathcal{L}_{i j}^{n}=\frac{4^{i}}{\pi} \int_{-1}^{1} x^{n} Q_{i}(x) \frac{1}{\sqrt{1-x^{2}}}\left(\begin{array}{ll}
1 & x \\
x & 1
\end{array}\right) Q_{j}^{*}(x) d x,
$$

where $\mathcal{L}_{i j}^{n}$ stands for the $(i, j)$ block of the matrix $\mathcal{L}^{n}$. As is usual for birth-and-death processes, the indices $i, j$ run from 0 on.

In this way, as noticed in [10], one can compute the entries of the powers $\mathcal{L}^{n}$ with

Copyright (c) by SIAM. Unauthorized reproduction of this article is prohibited. 
$\mathcal{L}$ thought of as a pentadiagonal scalar matrix, namely

$$
\mathcal{L}=\left(\begin{array}{cccccc}
0 & \frac{1}{2} & 1 & 0 & & \\
\frac{1}{2} & 0 & 0 & 1 & 0 & \\
\frac{1}{4} & 0 & 0 & 0 & 1 & \ddots \\
& \frac{1}{4} & 0 & 0 & 0 & \ddots \\
& & \ddots & \ddots & \ddots & \ddots
\end{array}\right) .
$$

$\mathcal{L}$ is not a stochastic matrix since its rows do not add up to unity. Nevertheless, defining $\Delta$ to be the $2 \times 2$ block diagonal matrix with $\Delta_{i i}=2^{i} I$ for every block, we get from (3.3) that $\Delta \mathcal{L} \Delta^{-1} \Delta \Phi=x \Delta \Phi$ and thus if $P=\Delta \mathcal{L} \Delta^{-1}$ and $\widetilde{\Phi}=\Delta \Phi$, we have $P \widetilde{\Phi}=x \widetilde{\Phi}$. The scalar version of $P$ is now the stochastic matrix

$$
P=\left(\begin{array}{cccccc}
0 & \frac{1}{2} & \frac{1}{2} & 0 & & \\
\frac{1}{2} & 0 & 0 & \frac{1}{2} & 0 & \\
\frac{1}{2} & 0 & 0 & 0 & \frac{1}{2} & \ddots \\
& \frac{1}{2} & 0 & 0 & 0 & \ddots \\
& & \ddots & \ddots & \ddots & \ddots
\end{array}\right) .
$$

Observe that the norm of $\widetilde{Q}_{n}$, defined in (3.1), satisfies $\left\|\widetilde{Q}_{n}\right\|^{2}=\pi$. This is nothing but the example considered at the end of [20] in the special case of $p=q=1 / 2$.

In the last few years a number of new families of matrix valued orthogonal polynomials have been computed explicitly along with their orthogonality measure. Typically they are joint eigenfunctions of some fixed differential operator with matrix coefficients. This search was initiated in [5], but nontrivial examples were not discovered until $[6]$ and $[13,16]$. The family of examples we will consider later is related to one of these examples and is obtained by modifying the situation discussed in [30].

4. Quasi-birth-and-death processes. Now we come to the last character of our story. For our purposes, we consider a two dimensional Markov chain with discrete time. The state space consists of the pair of integers $(i, j), i \in\{0,1,2, \ldots\}, j \in$ $\{1, \ldots, d\}$. The first component is usually called the level and the second one the phase. The one-step transition probability matrix, which we will denote (as before) by $P$ has a block tridiagonal structure (see (4.2)). This indicates that in one unit of time a transition can change the phase without changing the level, or can change the level (and possibly the phase) to either of the adjacent levels. The probability of going in one step from state $(i, j)$ to state $\left(i^{\prime}, j^{\prime}\right)$ is given by the $\left(j, j^{\prime}\right)$ element of the block $P_{i, i^{\prime}}$. Clearly in the case when the number of phases $d$ is one we are back to the case of an ordinary birth-and-death process. In general, these processes are known as (discrete time) quasi-birth-and-death processes.

For a much more detailed presentation of this field, as well as its connections with queueing problems in network theory as well as the general field of communication systems, the reader should consult $[24,27]$ and some of the references in [3].

Once one has the notions introduced in the previous sections it is very natural to connect them and to analyze these interesting Markov chains in terms of the 
corresponding spectral properties of the resulting family of matrix valued orthogonal polynomials. As pointed out before one can see the seed for this in the work of Krel̆n, as well as in the original paper [20].

We have identified two references where the corresponding Karlin-McGregor representation formula has been explicitly given, but there may be others since this is such a natural extension of the scalar theory; see [3] and [10]. In the case of quasibirth-and-death processes one replaces (2.2) by

$$
P_{i j}^{n}=\left(\int x^{n} Q_{i}(x) W(x) Q_{j}^{*}(x) d x\right)\left(\int Q_{j}(x) W(x) Q_{j}^{*}(x) d x\right)^{-1} .
$$

A different but related path to this circle of ideas in connection with network models can be seen in [1].

In [3] one finds some interesting examples where this representation formula is computed explicitly, including a new derivation of the result dealing with the case of random walk on the integers. In [10] one finds an example, taken from [9], where the observation is made that one has a stochastic matrix. The family of examples to be considered in the following sections is an extension of this example.

Given the block transition probability matrix $P$, the problem of computing an invariant distribution row vector, i.e., a vector with nonnegative entries $\pi_{j}^{i}$,

$$
\boldsymbol{\pi}=\left(\boldsymbol{\pi}^{0} ; \boldsymbol{\pi}^{1} ; \ldots\right) \equiv\left(\pi_{1}^{0}, \pi_{2}^{0}, \ldots, \pi_{d}^{0} ; \pi_{1}^{1}, \pi_{2}^{1}, \ldots, \pi_{d}^{1} ; \ldots\right)
$$

such that

$$
\boldsymbol{\pi} P=\boldsymbol{\pi}
$$

leads to a complicated system of equations.

If

$$
P=\left(\begin{array}{ccccc}
B_{0} & A_{0} & & & \\
C_{1} & B_{1} & A_{1} & & \\
& C_{2} & B_{2} & A_{2} & \\
& & \ddots & \ddots & \ddots
\end{array}\right),
$$

we have

$$
\pi^{0} B_{0}+\pi^{1} C_{1}=\pi^{0}
$$

and then, for $n \geq 1$,

$$
\boldsymbol{\pi}^{n-1} A_{n-1}+\boldsymbol{\pi}^{n} B_{n}+\boldsymbol{\pi}^{n+1} C_{n+1}=\boldsymbol{\pi}^{n} .
$$

This gives, as is easy to check, the rather unpleasant expressions

$$
\begin{gathered}
\boldsymbol{\pi}^{1}=\boldsymbol{\pi}^{0}\left(I-B_{0}\right) C_{1}^{-1}, \\
\boldsymbol{\pi}^{2}=\boldsymbol{\pi}^{0}\left[\left(I-B_{0}\right) C_{1}^{-1}\left(I-B_{1}\right)-A_{0}\right] C_{2}^{-1}, \\
\boldsymbol{\pi}^{3}=\boldsymbol{\pi}^{0}\left[\left(I-B_{0}\right) C_{1}^{-1}\left(I-B_{1}\right) C_{2}^{-1}\left(I-B_{2}\right)-A_{0} C_{2}^{-1}\left(I-B_{2}\right)-\left(I-B_{0}\right) C_{1}^{-1} A_{1}\right] C_{3}^{-1} .
\end{gathered}
$$

These formulas require that the matrices $C_{n}$ be invertible. Under these conditions, one can derive nicer looking expressions for the invariant distribution (see [23]). There are 
many issues here that we leave untouched in this analysis. For instance, the possibility of choosing $\boldsymbol{\pi}^{0}$ with nonnegative entries so that all the subsequent $\boldsymbol{\pi}^{n}$ will have this property requires extra conditions.

The general theory of quasi-birth-and-death processes is not restricted to the case when the matrices $A_{n}, B_{n}$, and $C_{n}$ are all square matrices of the same size and $A_{n}$ and $C_{n}$ are nonsingular. It remains an interesting challenge to find a mathematical setup that can accommodate such a situation.

Now that we have seen how to extend the Karlin-McGregor representation formula to the block tridiagonal case it is important to get examples where the polynomials and the orthogonality matrix can be explicitly written down. This is the purpose of the next three sections.

5. Matrix valued spherical functions and matrix valued orthogonal polynomials. The theory of matrix valued spherical functions, initially discussed in [34], is one of the routes leading to explicit families of matrix valued orthogonal polynomials and their orthogonality measure. The first family of examples appeared in $[13,16]$ in connection with $G=\mathrm{SU}(3)$. The size of the matrices here is already arbitrary, and the orthogonality matrix has a scalar factor of the form $x^{\alpha}(1-x)$. The extension to the case where this scalar factor can be taken to be $x^{\alpha}(1-x)^{\beta}$ for arbitrary $\alpha, \beta>-1$ was undertaken in [9] in the $2 \times 2$ case without any reference to group representation theory. Further examples of this kind are given in [14]. The role of group representation theory in getting away from the $2 \times 2$ case can be seen in [15]. Finally, [30] displays for the case of $G=\mathrm{SU}(N+1)$ families of orthogonal polynomials depending on three parameters, $\alpha, \beta$, and $k$. In the special case of $k=\frac{\beta+1}{2}$, one recovers the results of [9].

These orthogonal polynomials are given by properly "packaged and conjugated" sets of matrix valued spherical functions. These spherical functions correspond to irreducible representations of $\mathrm{U}(N)$ and therefore are parameterized by partitions

$$
\mu=\left(m_{1}, m_{2}, \ldots, m_{N}\right) \in \mathbb{Z}^{N} \quad \text { such that } \quad m_{1} \geq m_{2} \geq \cdots \geq m_{N} .
$$

In this paper, following [30], we use only "one step" representations given by a partition

$$
\mu=(\underbrace{m+\ell, \ldots, m+\ell}_{k}, \underbrace{m, \ldots, m}_{N-k}), \quad 1 \leq k \leq N-1 .
$$

In terms of the parameters $\alpha$ and $\beta$, one has $\alpha=m$ and $\beta=N-1$. The remaining free parameter $\ell$ will determine the size of the corresponding matrix valued orthogonal polynomials and is independent of $N$. In the next section it will be related to the parameter $d$ appearing in [3].

The examples that have been worked out so far indicate that the matrix valued orthogonal polynomials that result from matrix valued spherical functions lead to a block tridiagonal matrix that can be made into a stochastic one. This will be seen, for our family of examples, in section 8 .

Although it is possible to obtain examples of stochastic matrices arising in a different fashion, see, for instance [2], we are not aware of any other general scheme that would produce these desirable kinds of matrices in a systematic fashion.

6. A family of examples arising from the complex projective space. In what follows we shall use $E_{i j}$ to denote the matrix with entry $(i, j)$, which is equal to 1 and 0 elsewhere, where the indices $i, j$ run from 0 on. 
Let $d \in\{1,2,3, \ldots\}, \alpha, \beta>-1$ and $0<k<\beta+1$. From [30] we have that the differential operator

$$
D=x(1-x) \frac{d^{2}}{d x^{2}}+(C-x U) \frac{d}{d x}+V,
$$

with matrix coefficients given by

$$
\begin{gathered}
C=\sum_{i=0}^{d-1}(\alpha+d-i) E_{i i}-\sum_{i=0}^{d-2}(i+1) E_{i+1, i}, \quad U=\sum_{i=0}^{d-1}(\alpha+\beta+d+i+1) E_{i i}, \\
V=-\sum_{i=0}^{d-1} i(\alpha+\beta-k+i+1) E_{i i}+\sum_{i=0}^{d-2}(d-i-1)(\beta-k+i+1) E_{i, i+1},
\end{gathered}
$$

admits as eigenfunctions (the conjugates of) a family of orthogonal polynomials with respect to the $d \times d$ weight function

$$
W(x)=x^{\alpha}(1-x)^{\beta} Z(x), \quad x \in[0,1],
$$

where

$$
Z(x)=\sum_{i, j=0}^{d-1}\left(\sum_{r=0}^{d-1}\left(\begin{array}{l}
r \\
i
\end{array}\right)\left(\begin{array}{l}
r \\
j
\end{array}\right)\left(\begin{array}{c}
d+k-r-2 \\
d-r-1
\end{array}\right)\left(\begin{array}{c}
\beta-k+r \\
r
\end{array}\right)(1-x)^{i+j} x^{d-r-1}\right) E_{i j} .
$$

Initially the value of $k$ is an integer, but (with the appropriate notation) this can be taken to be any value in the range indicated above. Likewise, in the group theoretical setup of [30] one first takes $\alpha$ and $\beta$ to be integers but then observes that this holds for $\alpha$ and $\beta$ as above.

In the language of [14], $\{W, D\}$ is a classical pair.

In [28] one finds an explicit expression for a family of eigenfunctions of $D$ in terms of the matrix valued hypergeometric function ${ }_{2} F_{1}$ introduced in [35].

In principle it is possible to obtain the coefficients for the three term recursion relation satisfied by any family of orthogonal polynomials whose existence is proved in [30]. We have not done this, but instead, since our goal is to obtain a family of matrix valued orthogonal polynomials $\left\{Q_{n}(x)\right\}_{n \geq 0}$ with specific probabilistic properties, we modify appropriately the classical pair in [30]. This is the goal of the next section.

Remark. Notice that the notation in $[13,14,15,16,30]$ and the one in [3] (which we follow here) are related by $d=\ell+1$.

7. The new equivalent classical pair. Let us consider the following nonsingular upper triangular matrix:

$$
T=\sum_{i \leq j}(-1)^{i} \frac{(-j)_{i}}{(1-d)_{i}} \frac{(\alpha+\beta-k+j+1)_{i}}{(\beta-k+1)_{i}} E_{i j},
$$

where $(a)_{n}$ will denote the Pochhammer symbol defined by $(a)_{n}=a(a+1) \cdots(a+n-1)$ for $n>0,(a)_{0}=1$. The purpose of choosing $T$ as above will be made clear below.

Let us consider the new classical pair $\{\widetilde{W}, \widetilde{D}\}$, where

$$
\widetilde{W}=T^{*} W T
$$

Copyright (C) by SIAM. Unauthorized reproduction of this article is prohibited. 
and

$$
\widetilde{D}=T^{-1} D T=x(1-x) \frac{d^{2}}{d x^{2}}+(\widetilde{C}-x \widetilde{U}) \frac{d}{d x}+\widetilde{V},
$$

where

$$
\begin{aligned}
\widetilde{C}= & \sum_{i=0}^{d-1}\left(\alpha+d-i+\frac{i(d-i)(\beta-k+i)}{\alpha+\beta-k+2 i}-\frac{(i+1)(d-i-1)(\beta-k+i+1)}{\alpha+\beta-k+2 i+2}\right) E_{i i} \\
& +\sum_{i=0}^{d-2}\left(1+i+\frac{(i+1)(d-i-2)(\beta-k+i+2)}{\alpha+\beta-k+2 i+3}\right. \\
& \left.\quad-\frac{(i+1)(d-i-1)(\beta-k+i+1)}{\alpha+\beta-k+2 i+2}\right) E_{i, i+1} \\
& +\sum_{i=0}^{d-2}\left(\frac{(i+1)(d-i-1)(\beta-k+i+1)}{\alpha+\beta-k+2 i+2}-\frac{i(d-i-1)(\beta-k+i+1)}{\alpha+\beta-k+2 i+1}\right) E_{i+1, i}, \\
\widetilde{U}= & \sum_{i=0}^{d-1}(\alpha+\beta+d+i+1) E_{i i}+\sum_{i<j}\left((-1)^{j-i}(i+1)_{j-i} \frac{\alpha+\beta-k+2 i+1}{(\alpha+\beta-k+i+1)_{j-i}}\right) E_{i j}, \\
\widetilde{V}= & -\sum_{i=0}^{d-1} i(\alpha+\beta-k+i+1) E_{i i} .
\end{aligned}
$$

The pair $\{\widetilde{W}, \widetilde{D}\}$ is equivalent to $\{W, D\}$ according to the definitions in [14]. Note that $T$ is chosen so that $\widetilde{V}$ turns out to be diagonal.

We now produce a particular family of polynomials $\left\{Q_{n}(x)\right\}_{n \geq 0}$ satisfying $Q_{0}(x)=$ $I$ and $\widetilde{D} Q_{n}^{*}(x)=Q_{n}^{*}(x) \Lambda_{n}$ with

$$
\Lambda_{n}=-\sum_{i=0}^{d-1}\left(n^{2}+(\alpha+\beta+d+i) n+i(\alpha+\beta-k+i+1)\right) E_{i i} .
$$

If we put $Q_{n}(x)=\sum_{j=0}^{n} A_{j}^{n} x^{j}$, we see that the leading coefficient $A_{n}^{n}$ can be conveniently chosen to be the lower triangular matrix

$$
\begin{aligned}
& \sum_{i \leq j}(-1)^{i+j}\left(\begin{array}{l}
j \\
i
\end{array}\right)(\alpha+\beta-k+2 i+1) \\
& \times \frac{(n)_{j-i}(k+n)_{d-j-1}(\alpha+\beta-k+n+j+1)_{i}(\alpha+\beta+n+d+j)_{n}}{(k)_{d-j-1}(\beta+d)_{n}(\alpha+\beta-k+i+1)_{j+1}} E_{j i},
\end{aligned}
$$

and that this determines $A_{j}^{n}$ for $j=n-1, n-2, \ldots, 0$. This is very similar to the analysis in section 3.1 of [11], where the final result is equations (3)-(5), expressing these polynomials in terms of the matrix valued hypergeometric function ${ }_{2} F_{1}$ of Tirao; see [35]. As mentioned in the last section, such an expression in terms of ${ }_{2} F_{1}$ is obtained in [28] for a family of polynomials that are related to $\left\{Q_{n}(x)\right\}_{n \geq 0}$ in the form $Q_{n}(x)=A_{n}^{n} T^{*}\left(\widetilde{A}_{n}^{n}\right)^{-1} P_{n}(x)\left(T^{*}\right)^{-1}$, where $P_{n}(x)=\widetilde{A}_{n}^{n} x^{n}+\cdots$. These $\left\{P_{n}(x)\right\}_{n \geq 0}$ do 
not satisfy the same recursion relation as ours. This choice of $A_{n}^{n}$ above is motivated by the remarkable fact that then our $\left\{Q_{n}(x)\right\}_{n \geq 0}$ satisfy

$$
Q_{n}(1) \mathbf{e}_{d}^{*}=\mathbf{e}_{d}^{*}
$$

where $\mathbf{e}_{d}$ is the $d$-dimensional row vector with all entries equal to 1 . In other words, the sum of the elements in each row of $Q_{n}(1)$ gives the value 1.

Below, we give the explicit expression for the stochastic block tridiagonal matrix going with the sequence $\left\{Q_{n}(x)\right\}_{n \geq 0}$.

THEOREM 7.1. The family of orthogonal polynomials introduced above satisfies the three term recursion relation

$$
x Q_{n}(x)=A_{n} Q_{n+1}(x)+B_{n} Q_{n}(x)+C_{n} Q_{n-1}(x), \quad n \geq 0,
$$

where $Q_{-1}(x)=0$ and $Q_{0}(x)=I$. For $n \geq 0, A_{n}$ is the lower bidiagonal matrix

$$
\begin{aligned}
A_{n} & =\sum_{i=0}^{d-1} \frac{(k+n)(\beta+n+d)(\alpha+\beta+n+d+i)(\alpha+\beta-k+n+i+1)}{(k+n+d-i-1)(\alpha+\beta-k+n+2 i+1)(\alpha+\beta+2 n+d+i)_{2}} E_{i i} \\
& +\sum_{i=0}^{d-2} \frac{(i+1)(k+n)(k+d-i-2)(\beta+n+d)}{(\alpha+\beta+2 n+d+i+1)(\alpha+\beta-k+n+2 i+3)(k+n+d-i-2)_{2}} E_{i+1, i},
\end{aligned}
$$

for $n \geq 1, C_{n}$ is the upper bidiagonal matrix

$$
\begin{aligned}
C_{n} & =\sum_{i=0}^{d-1} \frac{n(\alpha+n+i)(k+n+d-1)(\alpha+\beta-k+n+d+i)}{(k+n+d-i-1)(\alpha+\beta-k+n+2 i+1)(\alpha+\beta+2 n+d+i-1)_{2}} E_{i i} \\
& +\sum_{i=0}^{d-2} \frac{n(d-i-1)(k+n+d-1)(\beta-k+i+1)}{(\alpha+\beta+2 n+d+i)(\alpha+\beta-k+n+2 i+1)(k+n+d-i-2)_{2}} E_{i, i+1},
\end{aligned}
$$

Copyright $@$ by SIAM. Unauthorized reproduction of this article is prohibited. 
and for $n \geq 0, B_{n}$ is the tridiagonal matrix

$$
\begin{aligned}
B_{n}=\sum_{i=0}^{d-1}\left(1+\frac{n(k+n-1)(k+n+d-1)(\beta+n+d-1)}{(\alpha+\beta+2 n+d+i-1)(k+n+d-i-2)_{2}}\right. \\
\quad-\frac{(n+1)(k+n)(k+n+d)(\beta+n+d)}{(\alpha+\beta+2 n+d+i+1)(k+n+d-i-1)_{2}} \\
\quad+\frac{i(d-i)(k+d-i-1)(\beta-k+i)}{(\alpha+\beta-k+n+2 i)(k+n+d-i-1)_{2}} \\
\left.\quad-\frac{(i+1)(d-i-1)(k+d-i-2)(\beta-k+i+1)}{(\alpha+\beta-k+n+2 i+2)(k+n+d-i-2)_{2}}\right) E_{i i} \\
+\sum_{i=0}^{d-2} \frac{(d-i-1)(\beta-k+i+1)(\alpha+\beta-k+n+i+1)(\alpha+\beta+n+d+i)}{(k+n+d-i-1)(\alpha+\beta+2 n+d+i)(\alpha+\beta-k+n+2 i+1)_{2}} E_{i, i+1} \\
+\sum_{i=0}^{d-2} \frac{(i+1)(\alpha+n+i+1)(k+d-i-2)(\alpha+\beta-k+n+d+i+1)}{(k+n+d-i-2)(\alpha+\beta+2 n+d+i+1)(\alpha+\beta-k+n+2 i+2)_{2}} E_{i+1, i} .
\end{aligned}
$$

Proof. The tools required to prove these formulas are implicitly included in [12] and [29] for the special case of $\beta=k=1$. The detailed proof of these results can be obtained following the program described above.

The advantage of dealing with this equivalent classical pair $\{\widetilde{W}, \widetilde{D}\}$, normalized as above, shows up, for instance, in the fact that we have a stochastic matrix. The entries of $A_{n}, B_{n}$, and $C_{n}$ are nonnegative, and by applying both sides of the identity (7.2) to the vector $\mathbf{e}_{d}^{*}$, setting $x=1$, and using (7.1) we obtain that the sum of the entries in each row of our block tridiagonal matrix equals one.

In [31] one finds a nice and different proof for the fact that our Jacobi matrix is stochastic in the special case of $\beta=k=1$.

There are further advantages in dealing with the pair $\{\widetilde{W}, \widetilde{D}\}$ : an expression for the norms of the family $\left\{Q_{n}(x)\right\}_{n \geq 0}$ with respect to the matrix measure $\widetilde{W}$, defined in (3.1), is given by the diagonal matrix

$$
\begin{aligned}
\left\|Q_{n}\right\|_{\widetilde{W}}^{2}= & \left(Q_{n}, Q_{n}\right)_{\widetilde{W}}=\sum_{i=0}^{d-1}(-1)^{i} \frac{\Gamma(n+1) \Gamma(\beta+d) \Gamma(\alpha+n+i+1)(1-k-d-n)_{i}}{\left(\begin{array}{c}
d-1 \\
i
\end{array}\right) \Gamma(d) \Gamma(\alpha+\beta+d+i+2 n+1)} \\
& \times \frac{(k+d-i-1)_{n}(\alpha+\beta+d+i+n)_{n}(\alpha+\beta-k+i+n+1)_{d}}{(\alpha+\beta-k+2 i+n+1)(k)_{n}(\beta-k+1)_{i}(\beta+d)_{n}} E_{i i},
\end{aligned}
$$

where $\Gamma$ is the standard Gamma function. We point out that these matrix valued norms are given in our case by diagonal matrices, a fact that will play an important role later on.

For the benefit of the reader we include here the expression for all the quantities above in the case $d=1$. The weight $\widetilde{W}$ and differential operator $\widetilde{D}$ are given by

$$
\widetilde{W}(x)=x^{\alpha}(1-x)^{\beta}, \quad \widetilde{D}=x(1-x) \frac{d^{2}}{d x^{2}}+(\alpha+1+x(\alpha+\beta+2)) \frac{d}{d x} .
$$

Copyright (c) by SIAM. Unauthorized reproduction of this article is prohibited. 
Hence, we have that the coefficients of the three term recursion relation and the norms of the orthogonal polynomials are given by

$$
\begin{aligned}
A_{n} & =\frac{(n+\beta+1)(n+\alpha+\beta+1)}{(2 n+\alpha+\beta+1)(2 n+\alpha+\beta+2)}, \quad n \geq 0, \\
B_{n} & =1+\frac{n(n+\beta)}{2 n+\alpha+\beta}-\frac{(n+1)(n+\beta+1)}{2 n+\alpha+\beta+2}, \quad n \geq 0, \\
C_{n} & =\frac{n(n+\alpha)}{(2 n+\alpha+\beta)(2 n+\alpha+\beta+1)}, \quad n \geq 1, \\
\left\|Q_{n}\right\|_{\overparen{W}}^{2} & =\frac{\Gamma(n+1) \Gamma(n+\alpha+1) \Gamma(\beta+1)^{2}}{\Gamma(n+\beta+1) \Gamma(n+\alpha+\beta+1)(2 n+\alpha+\beta+1)}, \quad n \geq 0 .
\end{aligned}
$$

The polynomials $\left\{Q_{n}(x)\right\}_{n \geq 0}$ are exactly the Jacobi polynomials in the interval $[0,1]$, normalized to satisfy $Q_{n}(1)=1$.

The case $d=2$, for the special case of $k=\frac{\beta+1}{2}$, can be found in [9].

8. Probabilistic aspects of our family of examples. The corresponding Jacobi matrix

$$
P=\mathcal{L}=\left(\begin{array}{ccccc}
B_{0} & A_{0} & & & \\
C_{1} & B_{1} & A_{1} & & \\
& C_{2} & B_{2} & A_{2} & \\
& & \ddots & \ddots & \ddots
\end{array}\right)
$$

made up from the coefficients introduced in Theorem 7.1 is stochastic; that is, $P \mathbf{e}=\mathbf{e}$, where e denotes the semi-infinite column vector with all entries equal to 1 . Therefore, it gives a block tridiagonal transition probability matrix depending on three parameters, $\alpha, \beta$, and $k$.

The Markov process that results from $P$ is irreducible and aperiodic. Indeed, one can see that for any pair of states $(i, j),\left(i^{\prime}, j^{\prime}\right)$, every entry in the $\left(i, i^{\prime}\right)$-block of $P^{n}$ is positive if $n$ is large enough.

THEOREM 8.1. The Markov process that results from $P$ is never positive recurrent. If $-1<\beta \leq 0$, then the process is null recurrent. If $\beta>0$, then the process is transient.

Proof. One can use directly the Karlin-McGregor representation formula (4.1) to obtain Corollary 4.1 of [3]. The process turns out to be recurrent if and only if

$$
e_{j}^{T}\left(\int_{0}^{1} \frac{\widetilde{W}(x)}{1-x} S_{0}^{-1} d x\right) e_{j}=\infty
$$

for some $j \in\{1, \ldots, d\}$, where $e_{j}^{T}=(0, \ldots, 0,1,0, \ldots, 0)$ denotes the $j$ th unit vector and $S_{0}=\int_{0}^{1} \widetilde{W}(x) d x=\left\|Q_{0}\right\|_{\widetilde{W}}^{2}$ is the first moment. Otherwise, the process is transient. The explicit expression of the weight matrix in our case is $\widetilde{W}(x)=$ $x^{\alpha}(1-x)^{\beta} \widetilde{Z}(x)$, where $\widetilde{Z}(x)$ is a matrix polynomial and a detailed look shows that

$$
\widetilde{Z}(1)=\frac{(\beta+1)_{d-1}}{(d-1) !} \sum_{i, j=0}^{d-1} E_{i j} .
$$

Hence, condition (8.2) holds if and only if $-1<\beta \leq 0$.

Copyright (c) by SIAM. Unauthorized reproduction of this article is prohibited. 
Corollary 4.2 of [3] gives a necessary and sufficient condition for a process to be positive recurrent. This happens exactly when one of the entries of the measure $\widetilde{W}$ has a jump at the point 1 . But this is not true in our case given the form of $\widetilde{W}$ indicated above. So our process is never positive recurrent. This means that for $-1<\beta \leq 0$ the process is null recurrent.

All the considerations above result from explicit expressions for $\widetilde{W}(x)$ and the entries of $P$.

Now we come to a very delicate issue: the explicit computation of an invariant distribution. As noticed in (7.3), our matrix valued orthogonal polynomials $\left\{Q_{n}(x)\right\}_{n \geq 0}$ have the remarkable property that their norms are diagonal matrices. This provides us, for each $n$, with $d$ scalars which could be used (inspired by the case of birth-anddeath processes) as a way of getting an invariant row vector

$$
\boldsymbol{\pi}=\left(\boldsymbol{\pi}^{0} ; \boldsymbol{\pi}^{1} ; \ldots\right)
$$

with the property that

$$
\pi P=\pi
$$

Thus, we find the remarkable fact that the components of $\boldsymbol{\pi}$ can be computed by the recipe

$$
\boldsymbol{\pi}^{n}=\mathbf{e}_{d}\left(\left\|Q_{n}\right\|_{\widetilde{W}}^{2}\right)^{-1}, \quad n \geq 0
$$

where $\mathbf{e}_{d}$ is the $d$-dimensional row vector with all entries equal to 1 . The fact that the process is never positive recurrent implies that there exists no invariant distribution such that $\pi \mathrm{e}<\infty$.

The unicity of the invariant distribution $\pi$ holds as a consequence of the extended Perron-Frobenius theorem for countable nonnegative matrices (see [32, Theorem 5.4]), when the process is recurrent, i.e., $-1<\beta \leq 0$. However, we have extensive numerical evidence that this is true for all values of $\beta>-1$.

The case of random walk on the integers with general values of $p$ and $q(p+q=1)$ treated in [20] gives (for $p \neq q$ ) an example of a transient process where the invariant distribution is not unique. As mentioned above, it appears that even for values of $\beta$ when our process is transient we still have a unique invariant distribution which can be computed explicitly in terms of the norms of our orthogonal polynomials.

To conclude this section we exhibit the network associated with our family of examples. The states of our network are labeled (as in any two dimensional situation) by two indices $i=0,1,2, \ldots$ and $j=1,2, \ldots, d$. However, to write down a one-step transition probability matrix, one needs to agree on some linear order for the states. We use the convenient ordering

$$
(0,1),(0,2), \ldots,(0, d),(1,1),(1,2), \ldots,(1, d),(2,1),(2,2), \ldots,(2, d), \ldots
$$

so that, for instance, the label 3 in the following graph refers to $(0,3)$, while the label $d+2$ refers to $(1,2)$, etc. This choice of lexicographic order in one of the components and then in the other is an unpleasant feature that cannot be avoided completely. 
The state space and the corresponding one-step transitions appear as follows:

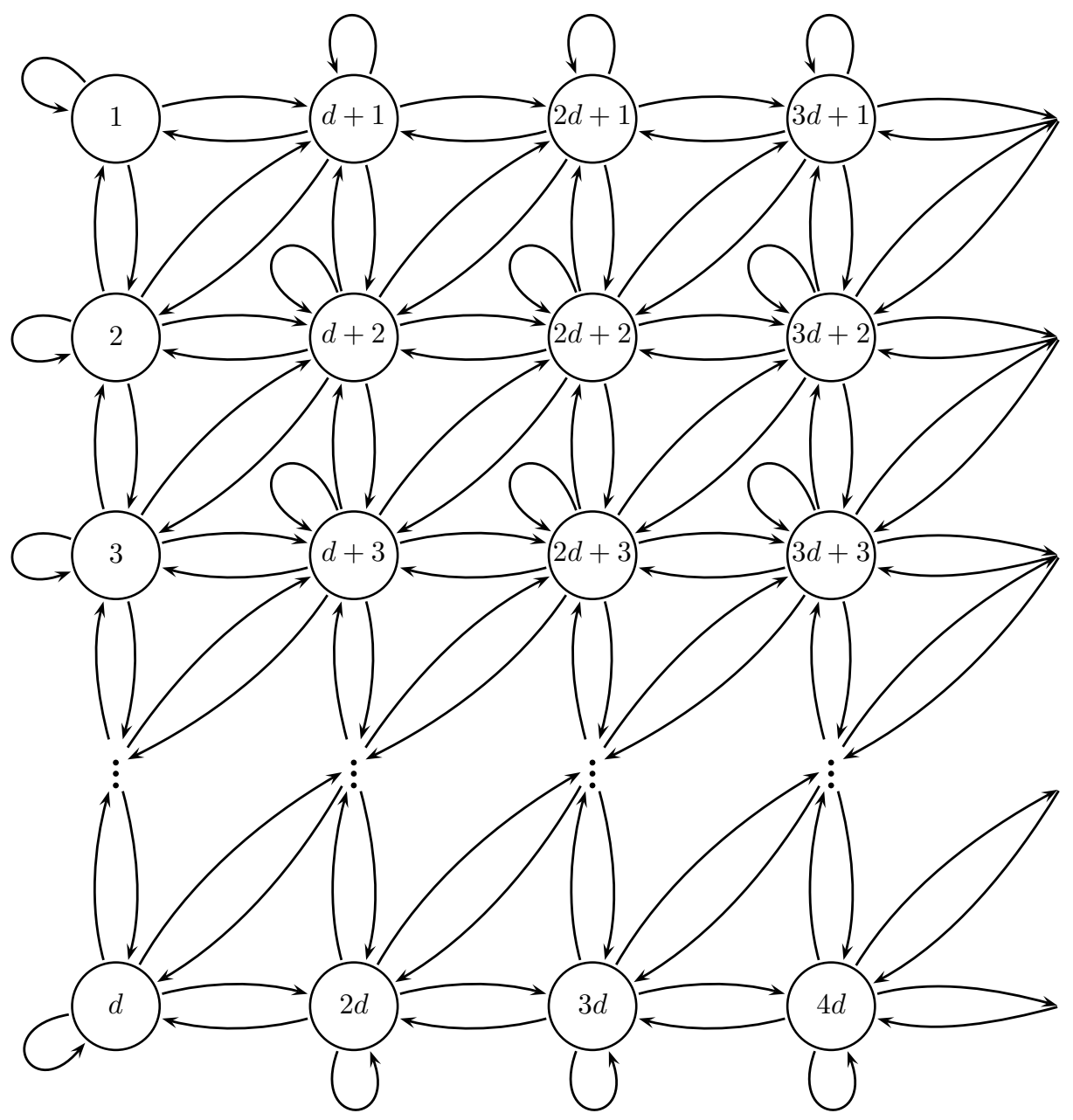

9. The shape of the invariant distribution. In this section we will study in more detail the behavior of the invariant distribution when the number of phases $d$ is equal to two, a luxury we can afford since we have an analytic expression. In this case, the associated network takes the form

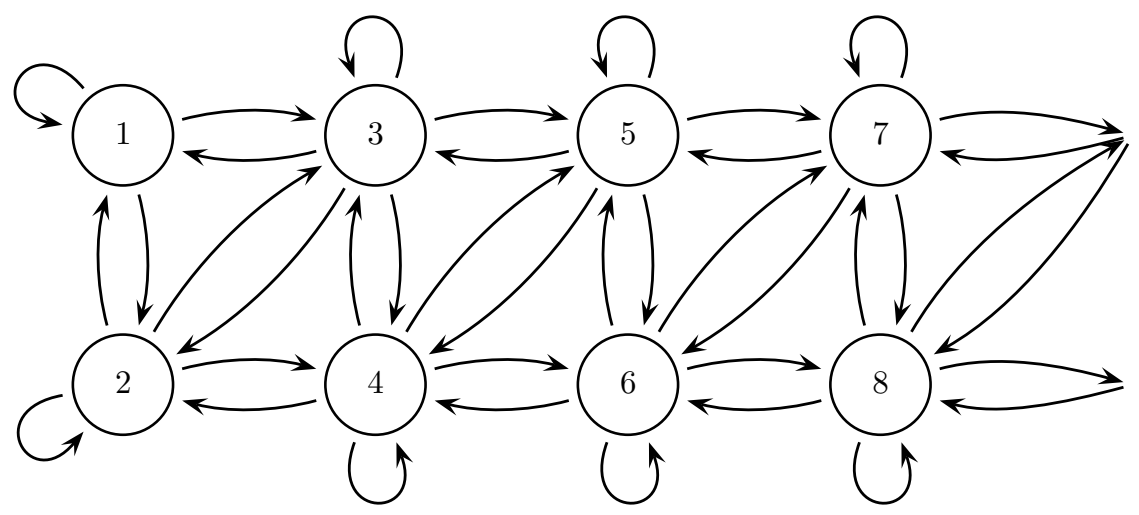

Copyright (C) by SIAM. Unauthorized reproduction of this article is prohibited. 


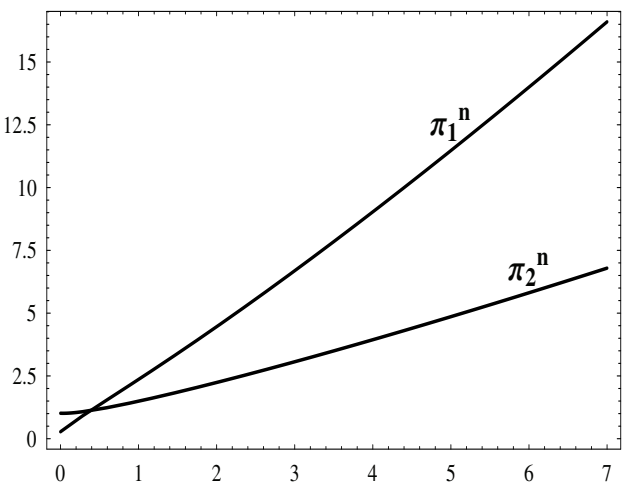

FIG. 9.1. $\alpha=-0.9, \beta=0.1, k=0.8$.

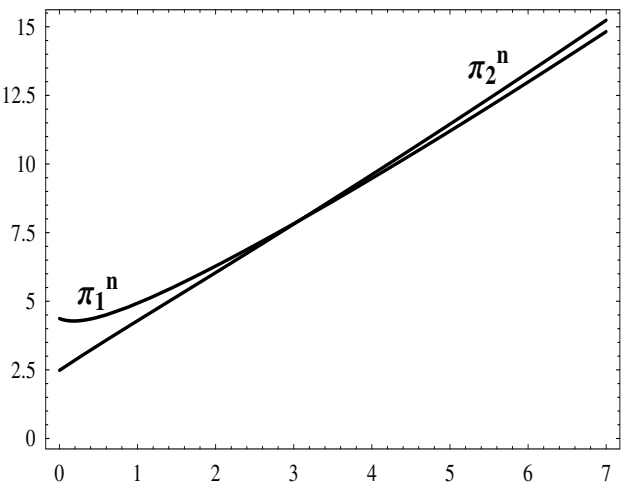

FIG. 9.2. $\alpha=2.5, \beta=0.1, k=0.55$.

The invariant distribution $\boldsymbol{\pi}$ such that $\boldsymbol{\pi} P=\boldsymbol{\pi}$ is given by

$$
\boldsymbol{\pi}=\left(\boldsymbol{\pi}^{0} ; \boldsymbol{\pi}^{1} ; \ldots\right),
$$

where $\boldsymbol{\pi}^{n}, n \geq 0$, is the 2 -dimensional vector given by

$$
\begin{aligned}
\boldsymbol{\pi}^{n}= & \frac{\Gamma(n+\alpha+\beta+2) \Gamma(n+\beta+2)}{\Gamma(n+\alpha+1) \Gamma(n+1) \Gamma(\beta+2)^{2}(n+\alpha+\beta-k+2)} \\
& \left(\frac{k(2 n+\alpha+\beta+2)}{n+k}, \frac{(\beta-k+1)(2 n+\alpha+\beta+3)(n+\alpha+\beta+2)}{(n+\alpha+1)(n+k+1)}\right) .
\end{aligned}
$$

From this explicit expression we can easily obtain several quantities. Data of special interest may be the initial value and the asymptotic behavior. The initial value is given by

$$
\boldsymbol{\pi}^{0}=\frac{\Gamma(\alpha+\beta+3)}{\Gamma(\alpha+1) \Gamma(\beta+2)(\alpha+\beta-k+2)}\left(1, \frac{(\beta-k+1)(\alpha+\beta+3)}{(\alpha+1)(k+1)}\right) .
$$

The asymptotic behavior follows using asymptotic formulas for the Gamma function such as $\frac{\Gamma(z+\alpha)}{\Gamma(z)} \approx z^{\alpha}$ as $|z| \rightarrow \infty$. Hence, we have

$$
\lim _{n \rightarrow \infty} \pi^{n}=\left\{\begin{array}{clc}
(\infty, \infty) & \text { if } & \beta>-\frac{1}{2}, \\
\frac{4}{\pi}(2 k, 1-2 k) & \text { if } & \beta=-\frac{1}{2}, \\
(0,0) & \text { if } & -1<\beta<-\frac{1}{2} .
\end{array}\right.
$$

In what follows we shall include plots of the two components $\boldsymbol{\pi}_{1}^{n}$ and $\boldsymbol{\pi}_{2}^{n}$, as functions of $n$, in a few representative cases. The general shape of both curves can change depending on the values of the parameters $\alpha, \beta$, and $k$. A look at the role of the parameter $\beta$ gives rise to four regions, namely $-1<\beta<-1 / 2, \beta=-1 / 2,-1 / 2<$ $\beta<0$, and $\beta \geq 0$. The parameter $\alpha$ only has influence on cosmetic changes like curvature and initial values depending on $-1<\alpha<0$ or $\alpha \geq 0$, while $k$ affects the shape of the plots when its value is the middle point $\frac{\beta+1}{2}$ of its possible range and the situation in the rest of values is quite symmetric.

Figures 9.1 and 9.2 show the most interesting situations when $\beta>0$.

Figures 9.3 and 9.4 show how the situation can change for small perturbations around $\beta=-1 / 2$. In Figure 9.3 both curves have a logarithmic growth and the second component has a minimum, while in Figure 9.4 both curves tend to 0 . 


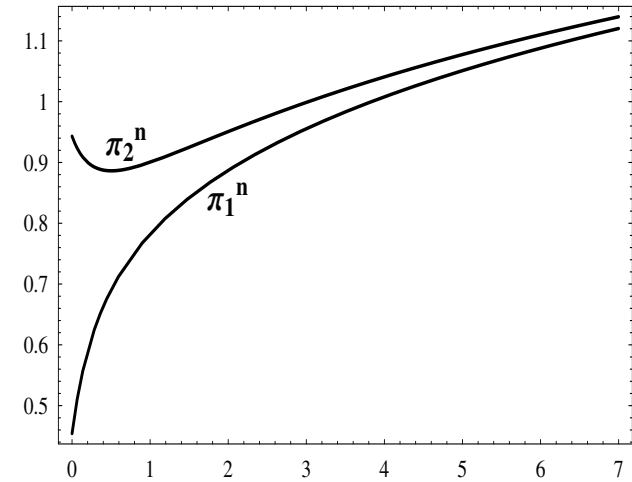

FIG. 9.3. $\alpha=-0.8, \beta=-0.4, k=0.3$.

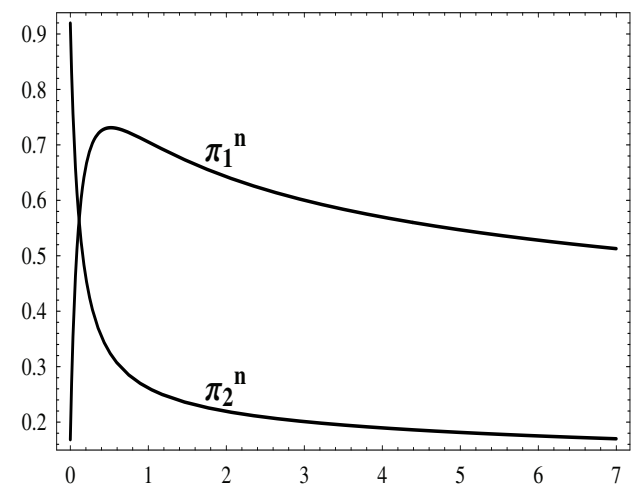

FIG. 9.5. $\alpha=-0.98, \beta=-0.6, k=0.3$.

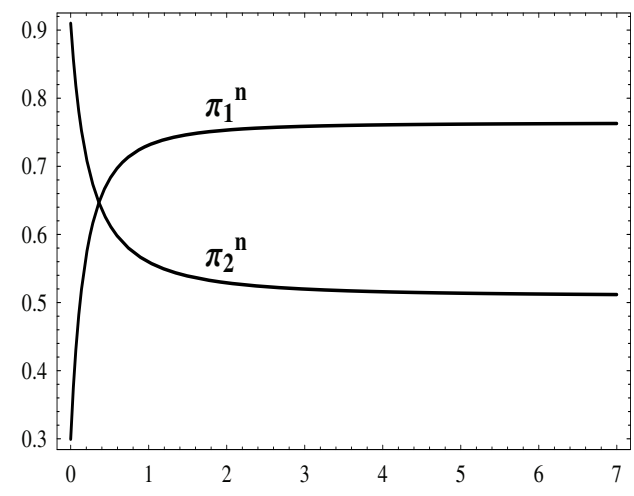

FIG. 9.7. $\alpha=-0.92, \beta=-0.5, k=0.3$.

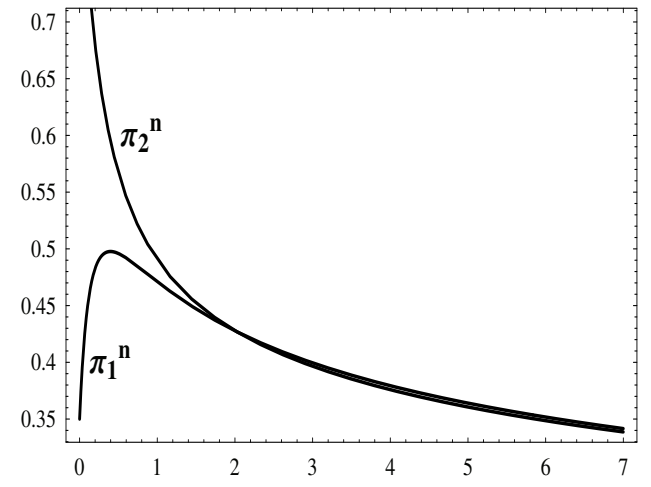

FIG. 9.4. $\alpha=-0.9, \beta=-0.6, k=0.2$.

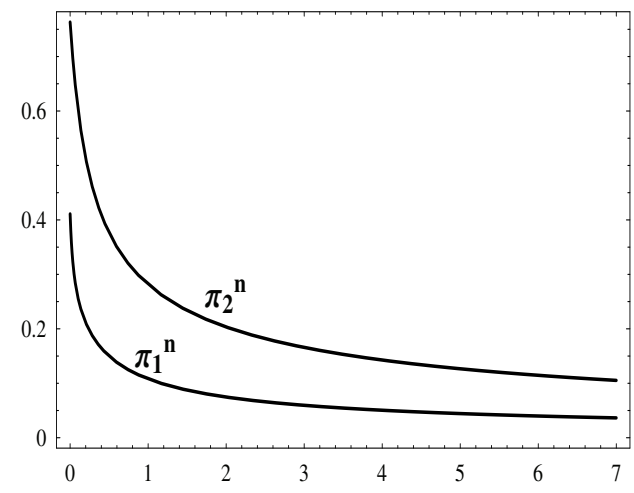

FIG. 9.6. $\alpha=-0.9, \beta=-0.8, k=0.05$.

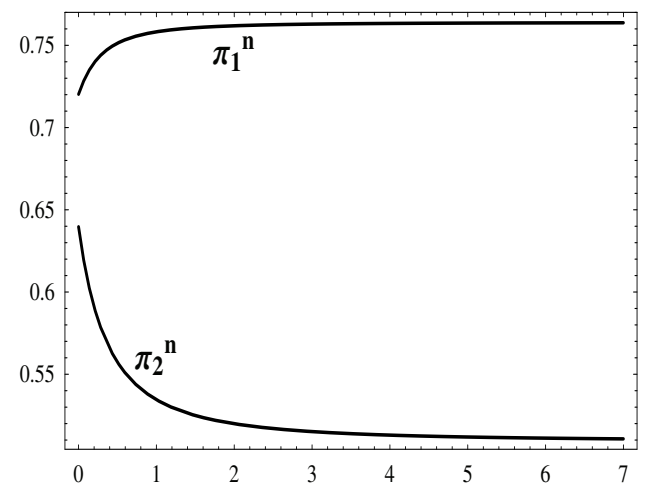

FIG. 9.8. $\alpha=-0.6857, \beta=-0.5, k=0.3$.

Copyright $@$ by SIAM. Unauthorized reproduction of this article is prohibited. 


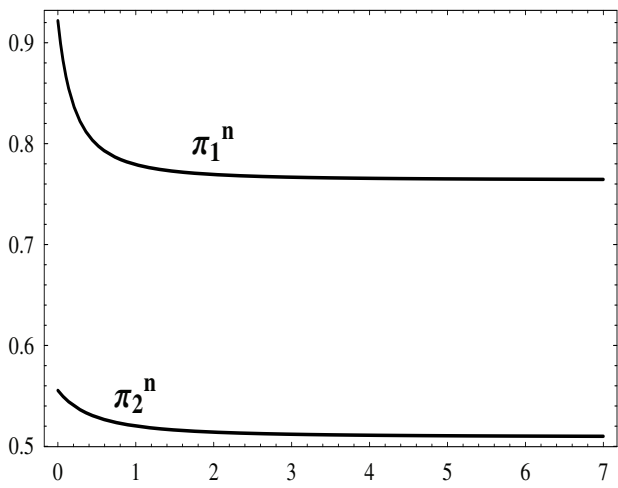

FIG. 9.9. $\alpha=-0.4857, \beta=-0.5, k=0.3$.

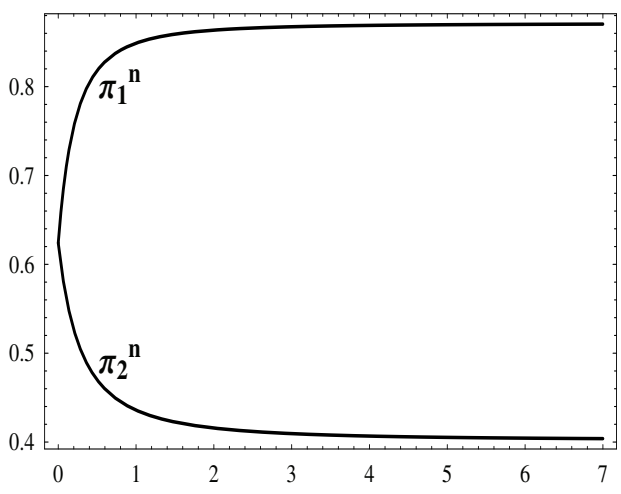

FIG. 9.11. $\alpha=-0.8, \beta=-0.5, k=0.3421$

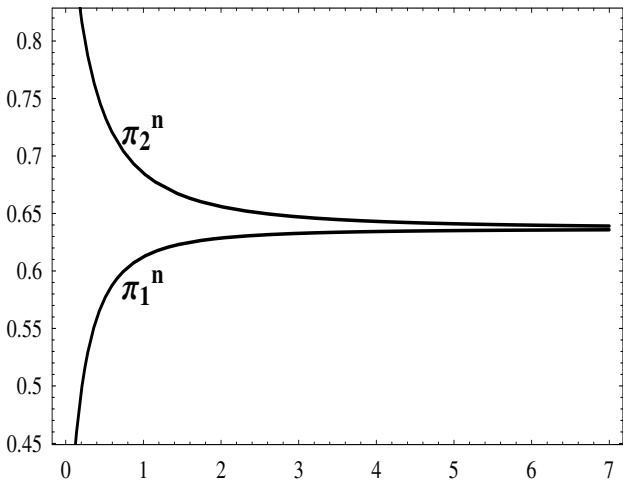

FIG. 9.10. $\alpha=-0.9, \beta=-0.5, k=0.25$.

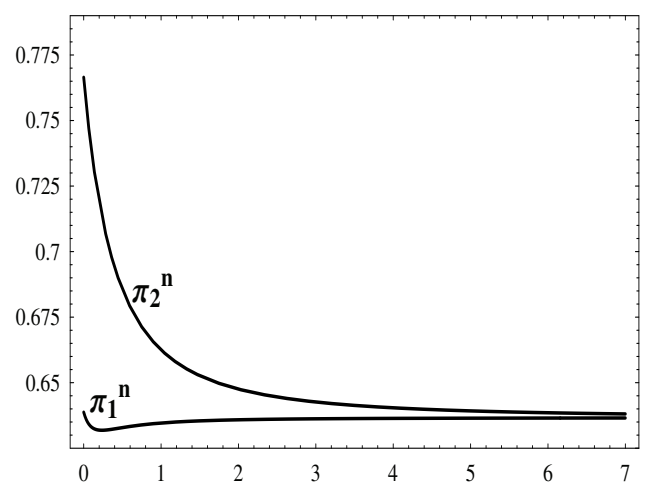

FIG. 9.12. $\alpha=-0.7, \beta=-0.5, k=0.25$.

We observe that Figure 9.5, with $k$ now approaching $\beta+1$, is similar to Figure 9.4. In Figure 9.6 we observe the consequences of $k$ being very small.

The remaining figures refer to the case $\beta=-1 / 2$, where both components converge for large $n$. In Figure 9.7 we observe that the initial value of the first component is always lower than that of the second component and that the initial value of the second component is always greater than that of the first component. A small change of the value of $\alpha$ has the effect that the first component is always greater than the second component, as we can see in Figure 9.8.

Small perturbations on $\alpha$ change the curvatures of the components in Figure 9.9 with respect to Figure 9.8. In Figure 9.10 both components tend to the same value without ever touching, a consequence of choosing $k=\frac{\beta+1}{2}$.

The last two figures show how the situation can change for small perturbations of $\alpha$ and $k$. In Figure 9.11 both curves start from the same value and then they converge to different limits, while in Figure 9.12 both components converge to the same limit.

10. Concluding remarks. A block tridiagonal matrix $\mathcal{L}$ with nonnegative entries and individual rows that add up to 1 gives rise to a quasi-birth-and-death process. The explicit evaluation of $\mathcal{L}^{n}$, for arbitrary $n=1,2,3, \ldots$, can be greatly simplified by using ideas that go back to Karlin and McGregor and have been explicitly set forth in $[3,10]$. The only major difficulty here is that of computing the weight matrix $W(x)$. In this paper we start from a rich group theoretical situation that yields $W(x)$ as well 
as a matrix $\mathcal{L}$ of the type envisaged previously. There are enough free parameters here to give instances of transient as well as recurrent Markov chains. It remains as interesting challenge to find some real life applications to this large collection of examples.

\section{REFERENCES}

[1] S. Basu And N. K. Bose, Matrix Stieltjes series and network models, SIAM J. Matrix Anal. Appl., 14 (1983), pp. 209-222.

[2] Ju. M. Berezans'KII, Expansions in Eigenfunctions of Selfadjoint Operators, Transl. Math. Monogr. 17, American Mathematical Society, Providence, RI, 1968.

[3] H. Dette, B. Reuther, W. J. Studden, And M. Zygmunt, Matrix measures and random walks with a block tridiagonal transition matrix, SIAM J. Matrix Anal. Appl., 29 (2006), pp. $117-142$.

[4] A. J. DurÁn, On orthogonal polynomials with respect to positive definite matrix of measures, Canad. J. Math., 47 (1995), pp. 88-112.

[5] A. J. DuRÁN, Matrix inner product having a matrix symmetric second order differential operator, Rocky Mountain J. Math., 27 (1997), pp. 585-600.

[6] A. J. Durán And F. A. GRÜnBaum, Orthogonal matrix polynomials satisfying second order differential equations, Int. Math. Res. Not., 10 (2004), pp. 461-484.

[7] W. FELler, On second order differential operators, Ann. of Math. (2), 61 (1955), pp. 90-105.

[8] I. J. Good, Random motion and analytic continued fractions, Math. Proc. Cambridge Philos. Soc., 54 (1958), pp. 43-47.

[9] F. A. Grünbaum, Matrix valued Jacobi polynomials, Bull. Sci. Math., 127 (2003), pp. 207-214.

[10] F. A. Grünbaum, Random walks and orthogonal polynomials: Some challenges, Probability, Geometry and Integrable Systems, Vol. 55, MSRI Publications, Cambridge University Press, Cambridge, UK, pp. 241-260.

[11] F. A. GRünbaum And M. D. De LA IGLesia, Matrix valued orthogonal polynomials related to $S U(N+1)$, their algebras of differential operators and the corresponding curves, Experiment. Math., 16 (2007), pp. 189-207.

[12] F. A. Grünbaum, I. Pacharoni, and J. A. Tirao, A matrix-valued solution to Bochner's problem, J. Phys. A: Math. Gen., 34 (2001), pp. 10647-10656.

[13] F. A. Grünbaum, I. Pacharoni, and J. A. Tirao, Matrix valued spherical functions associated to the complex projective plane, J. Funct. Anal., 188 (2002), pp. 350-441.

[14] F. A. Grünbaum, I. Pacharoni, and J. A. Tirao, Matrix valued orthogonal polynomials of the Jacobi type, Indag. Math. (N.S.), 14 (2003), pp. 353-366.

[15] F. A. Grünbaum, I. Pacharoni, and J. A. Tirao, Matrix valued orthogonal polynomials of Jacobi type: The role of group representation theory, Ann. Inst. Fourier (Grenoble), 55 (2005), pp. 2051-2068.

[16] F. A. Grünbaum, I. Pacharoni, and J. A. Tirao, An invitation to matrix valued spherical functions: Linearization of products in the case of the complex projective space $P_{2}(\mathbb{C})$, in Modern Signal Processing, D. Healy and D. Rockmore, eds., Cambridge University Press, Cambridge, UK, 2004, pp. 147-160.

[17] T. E. HARRIS, First passages and recurrence distributions, Trans. Amer. Math. Soc., 73 (1952), pp. $471-486$.

[18] M. E. H. Ismail, J. Letessier, D. Masson, and G. Valent, Birth and death processes and orthogonal polynomials, in Orthogonal Polynomials, P. Nevai, ed., Kluwer Academic Publishers, Dordrecht, The Netherlands, 1990, pp. 229-255.

[19] M. KAC, Random walk and the theory of Brownian motion, Amer. Math. Monthly, 54 (1947), pp. 369-391.

[20] S. Karlin And J. MCGregor, Random walks, IIlinois J. Math., 3 (1959), pp. 66-81.

[21] M. G. KREǏn, Fundamental aspects of the representation theory of Hermitian operators with deficiency index $(m, m)$, Amer. Math. Soc. Transl. Ser. 2, 97 (1971), Providence, RI, pp. $75-143$.

[22] M. G. KREINN, Infinite J-matrices and a matrix moment problem, Dokl. Akad. Nauk SSSR, 69 (1949), pp. 125-128.

[23] G. Latouche, C. E. M. Pearce, and P. G. Taylor, Invariant measures for quasi-birth-anddeath processes, Comm. Statist. Stochastic Models, 14 (1998), pp. 443-460.

[24] G. Latouche And V. Ramaswami, Introduction to Matrix Analytic Methods in Stochastic Modeling, ASA-SIAM Ser. Stat. Appl. Probab. 5, SIAM, Philadelphia, 1999. 
[25] W. Ledermann and G. E. Reuter, Spectral theory for the differential equations of simple birth and death processes, Philos. Trans. Roy. Soc. London, Ser. A., 246 (1954), pp. 321369.

[26] H. P. McKean, JR., Elementary solutions for certain parabolic partial differential equations, Trans. Amer. Math. Soc., 82 (1956), pp. 519-548.

[27] M. F. Neuts, Structured Stochastic Matrices of $M / G / 1$ Type and Their Applications, Marcel Dekker, New York, 1989.

[28] I. PaCharoni AND P. Román, A sequence of matrix valued orthogonal polynomials associated to spherical functions, Constr. Approx., 28 (2007), pp. 127-147.

[29] I. Pacharoni And J. A. TiRaO, Three term recursion relation for spherical functions associated to the complex projective plane, Math. Phys. Anal. Geom., 7 (2004), pp. 193-221.

[30] I. PaCharoni And J. A. TiRao, Matrix valued orthogonal polynomials arising from the complex projective space, Constr. Approx., 25 (2007), pp. 177-192.

[31] I. PAChARONI AND J. A. TIRAO, Three term recursion relation for spherical functions associated to the complex hyperbolic plane, J. Lie Theory, 17 (2007), pp. 791-828.

[32] E. Seneta, Non-negative Matrices and Markov Chains, 3rd ed., Springer-Verlag, New York, 2006.

[33] J. Shohat and J. Tamarkin, The Problem of Moments, American Mathematical Society Mathematical Surveys II, American Mathematical Society, Providence, RI, 1943.

[34] J. A. Tirao, Spherical functions, Rev. de la Unión Matem. Argentina, 28 (1977), pp. 75-98.

[35] J. A. Tirao, The matrix valued hypergeometric equation, Proc. Nat. Acad. Sci. USA, 100 (2003), pp. 8138-8141.

Copyright (c) by SIAM. Unauthorized reproduction of this article is prohibited. 